\title{
PITUITARY AND PARAPITUITARY TUMOURS* VALUE OF PERIMETRY IN DIAGNOSIS
}

\author{
BY \\ J. F. CULLEN \\ Department of Ophthalmology, University of Edinburgh
}

THE occurrence of pallor of the optic discs and bitemporal field defects suggests to every ophthalmologist the possibility of a pituitary tumour. This diagnosis is usually confirmed by $x$-ray examination of the skull, when the classical signs of ballooning of the sella turcica with backward displacement or erosion of the posterior clinoid processes and, in more advanced cases, gross enlargement of the pituitary fossa may be found. The significance of optic atrophy and bitemporal field defects when the sella turcica seemed normal in the roentgenogram was first emphasized by Cushing (1930) as suggestive of other tumours in this region, such as suprasellar meningiomata, craniopharyngiomata, gliomata, and aneurysms, and also of pituitary adenomata which may fail to enlarge the sella but spread upwards to involve the chiasm. McConnell and Mooney (1938) pointed out that, when a tumour is postchiasmal in situation, the optic discs may remain normal for a very long time, and Walsh (1957) mentions that normal discs may be seen in such circumstances even after the onset of complete blindness.

The significance of scotomatous field defects, unilateral in some cases, as a presenting sign of pituitary tumours has been re-emphasized by Kelly (1962). The typical progression of the field defect from the early loss in the upper temporal quadrants is clockwise in the right field and anti-clockwise in the left. When, however, a tumour spreads forwards to involve one optic nerve, there is often a temporal or nasal hemianopia in one field (depending upon whether the anterior angle of the chiasm is involved from the medial or lateral side) followed by blindness in the eye on this side, and the simultaneous or later appearance of a temporal field defect in the other (Scott, 1957); this was the position in both the patients mentioned below.

Negative plain $x$-ray findings, therefore, in suspected cases of pituitary tumour should not dissuade the ophthalmologist from this diagnosis, particularly in the presence of such suggestive perimetric evidence. Investigations by the neurologist and endocrinologist may be helpful but, unfortunately, are often unrevealing and, in such circumstances, more elaborate $x$-ray examinations such as angiography and air studies are needed to establish the diagnosis. Alfano, Almeida, and Whitworth (1963) reported two cases of far-advanced tumours, one a craniopharyngioma and the other a suprasellar meningioma, presenting with optic atrophy and irregular bitemporal field defects, where both plain skull $x$ rays and neurological examinations were normal. When, however, angiography was performed, there was shown to be unmistakable evidence of a space-occupying lesion in the region of the optic chiasm and, as a result, craniotomy was undertaken with gratifying results.

* Received for publication January 17, 1964. 
There are still some cases in which even angiography in addition to repeated plain $x$ rays of the sella may be normal and yet, on the evidence of the signs of involvement of the chiasmal region of the visual pathway, a tumour seems the most likely diagnosis. It is imperative in such cases, particularly with evidence of progressive loss of visual field and progressive visual failure, as in the patients reported in this communication, for the ophthalmologist to press his neurosurgical colleagues to explore the chiasmal region in order to exclude the presence of a tumour.

The following two case reports are presented, therefore, to emphasize some of these points. In both, unusual identical field defects were present. One patient showed optic atrophy and the other did not. Plain skull $x$-ray examination and angiography were performed in each case and proved negative but, with the evidence of progressive field loss (in one patient not until 4 years after the original investigation), air studies and dye ventriculography were performed, and eventually the chiasmal area was explored, and a large tumour was found and removed with improved visual function after operation.

\section{Case Reports}

Case 1, a 63-year-old postman, was referred to the Eye Department in September, 1962, because an optician could not improve his left eye with spectacles. He had complained of failing vision in the left eye for some months and thought his glasses needed alteration. He admitted, on questioning, that he had had some midfrontal headaches in the previous 3 months; these were, however, easily relieved by aspirin. He felt in full vigour and was still working on his daily rounds as a postman.

Examination.-The visual acuity was 6/12 and N5 in the right eye, but, with the left, he could only perceive hand movements. The left pupil reacted sluggishly to light, while the right pupil reacted normally. The fundi were normal and the optic discs well within normal limits as regards both colour and outline. The visual fields are shown in Fig. 1. There was a complete steep-edged temporal hemianopia in the right eye with macular sparing but, in the left, hand movements were perceived only in the temporal periphery.

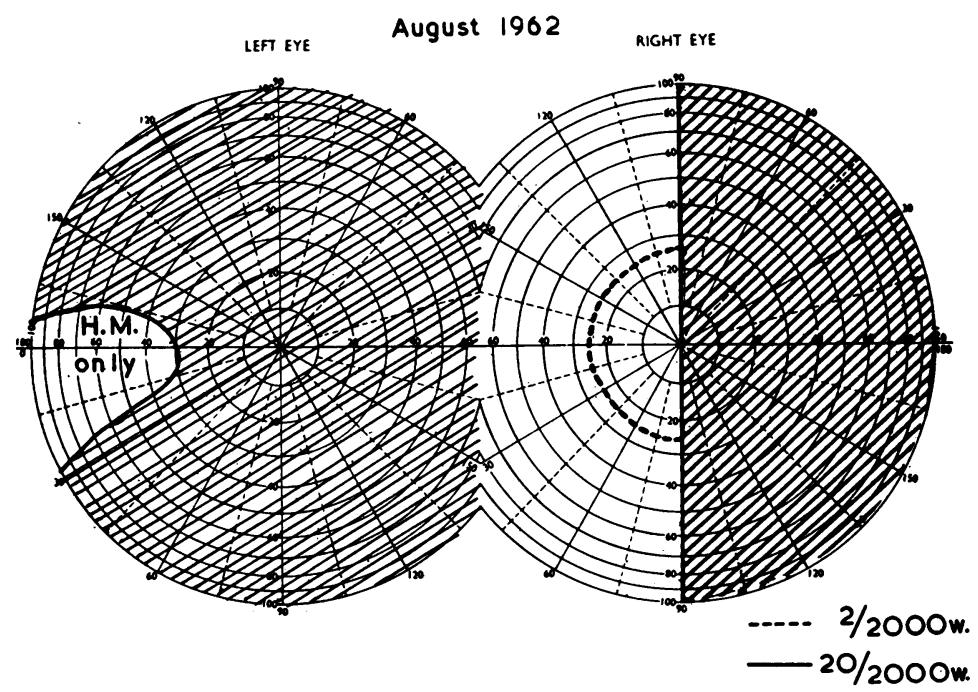

Fig. 1.-Case 1. Visual fields at first examination, September, 1962. Visual acuity: left eye, hand movements; right eye 56/12, N. 
The first possibility which occurred at this time was of a vascular lesion in the left carotid system, but there was no other evidence of generalized vascular disease and ophthalmodynanometry gave normal readings on both sides. In any event, if the left ophthalmic artery was involved, one would have expected the left disc to be atrophic. The other possibility was that of a tumour involving the chiasm for, on this alone, could one explain the findings on the basis of a single lesion, but, again, one would have expected such a tumour to be fairly far forward and optic atrophy would have been expected on the left side at least. Plain $x$ rays of the skull and optic foramina, and toned-down views of the sella were normal. The patient was then referred to the Neurosurgical Department for further investigation.

Repeated $x$ rays of the skull and chest were negative. Examinations of the blood, urine, and cerebrospinal fluid were unrevealing. Electro-encephalography showed a generalized abnormality on both sides, more marked on the left, and suggested a deep-seated lesion. Bilateral carotid arteriography showed no abnormality. A lumbar air encephalogram showed good filling of the basal cisterns but, again, this was reported as "within normal limits". During the course of these studies the patient complained of further gradual deterioration in vision and the left visual acuity dropped from $6 / 12$ to $6 / 36$.

With this further evidence of a progressive lesion, it was decided to carry the investigations even further. On November 16, 1962, air ventriculography was performed and there was a suggestion of a filling defect in the anterior part of the third ventricle. Myodil ventriculography was, therefore, thought necessary and these studies showed (Fig. 2) the presence of an expanding lesion in the anterior and inferior parts of the third ventricle.

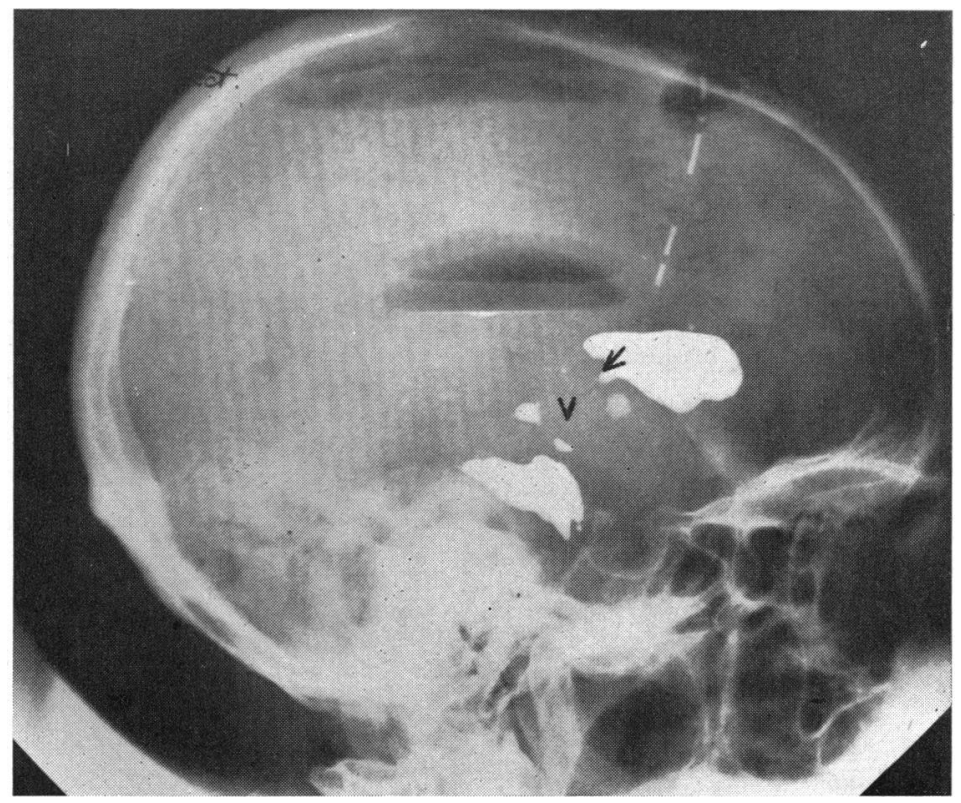

Fig. 2. Case 1. Myodil ventriculogram, showing filling defect in third ventricle (V) and arrow in foramen of Munro. The normal-sized sella is also seen.

Operation.-Exploration of the chiasmal region was therefore performed on November 26, 1962 , by Prof. F. J. Gillingham through a left frontal craniotomy. The operative findings were recorded as follows:

"There was found to be a dark tumour lifting and compressing the chiasm between both optic nerves but the left one more so. The left optic nerve was surrounded and distended by tumour and a small part of the tumour divided the nerve into two narrow bands. The tumour was very carefully excised and the optic nerves were quite free at the end of the procedure. The posterior portion of the tumour was not removed because of its extension upwards and backwards into the third ventricle." 
Pathological Examination.-The tumour was examined by Dr. A. Gordon of the Department of Neuropathology, who reported on it as follows:

"Histologically these are fragments of a cellular tumour of adenohypophyseal origin which shows moderate cellular pleomorphism and scanty mitoses. For the most part the cells are chromophobe but a considerable number of acidophils and a few basophils are identified. Whereas the latter may be surviving remnants of normal tissue, the former do appear to be rather more numerous and the cytology too abnormal to be explicable on this basis. In spite of the pleomorphism noted the appearances are those of a benign tumour rather than of a carcinoma (see Fig. 3) Chromophobe Adenoma (? ACidophil Differentiation)."

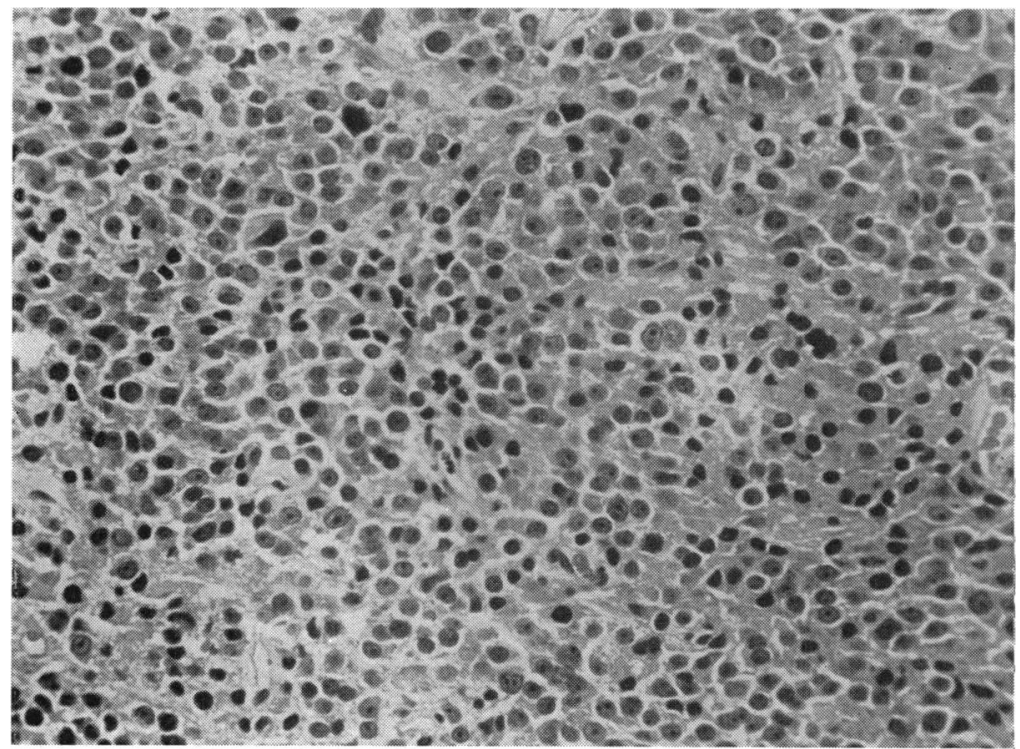

FIG. 3.-Case 1. Histology of pituitary adenoma, showing cellular pleomorphism.

$\times 234$.

Result.-The patient made an uneventful recovery after operation and a course of deep $x$-ray therapy was given to the pituitary region. The visual acuity improved rapidly after operation and by March, 1963, was 6/6, N5 in the right eye and 6/18, N18 in the left. The visual field had improved considerably in both eyes (Fig. 4) and the optic discs were still normal.

Fig. 4.-Case 1. Visual fields 4 months after operation. Visual acuity: left eye $6 / 18$, N18; right eye $6 / 6, \mathrm{~N} 5$.

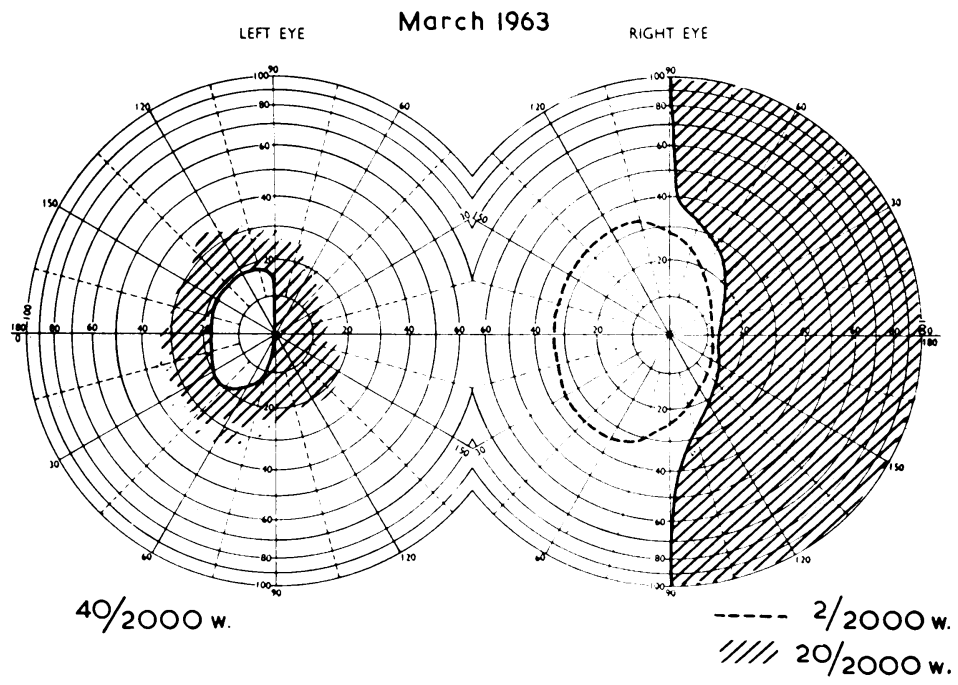


When the patient was last seen in July, 1963, the visual acuity had improved still further in the left eye to 6/9-2 and N6, and the visual field continued to expand slowly in each eye. There was now a suggestion of pallor of the left disc, but the right was still well within normal limits.

Comment.-The visual field recorded in this patient is not unusual in advanced cases of pituitary tumour where one optic nerve has been severely stretched by an upward and forward expansion of the tumour, yet the absence of optic atrophy was certainly unusual in such circumstances. The degree of recovery which took place in this severely stretched nerve is important in demonstrating that no such case is hopeless, even when the visual acuity is very severely impaired. The most important feature, however, is the extent to which the radiological investigations had to be carried before any evidence of a tumour was forthcoming-both arteriograms and air studies giving negative findings. It was the development of further visual loss in the patient's good eye which finally made it imperative to proceed with ventriculography and operation. It was only when the contrast medium (Myodil) was injected into the ventricles, however, that the presence of a tumour was confirmed and, at operation, this proved after all to be a chromophobe adenoma of the pituitary.

Case 2, a 40-year-old housewife, was first seen in the Eye Department in November, 1959, complaining of sudden loss of vision 10 days previously. She stated that, on getting up on the morning in question, she found that her right eye was practically blind and that the vision in the left eye was also below normal. She had not had any pain in the eyes or headaches at any time and, apart from her visual symptoms, she felt in good health. Her significant previous history was that she had been treated for hysteria in 1953 and she had been investigated in the Gynaecological Department 3 years previously for amenorrhea. This had come on suddenly and was classified as secondary and was put down to an early menopause. She had had a very unhappy married life and eventually separated from her husband in 1960.

Examination.-In November, 1959, the visual acuity in the right eye was perception of hand movements, and 6/24, N8 in the left. The right pupil reaction was impaired, the left being normal. There was a suggestion of temporal pallor of the right disc but the left disc was normal. The visual fields showed what appeared to be a left homonymous type of defect with loss of the temporal field of the left eye and fixation bisected. The nasal field of the right eye was absent to all targets and fixation was cut, but large targets were perceived in the temporal field. $X$ rays of the skull were normal and neurological examination entirely negative. With the possibility of a vascular lesion in mind, carotid arteriography was done and this showed some atheromatous changes in the anterior and middle cerebral arteries, so that a vascular disease process seemed possible despite her age.

6 months later the visual acuity in the left eye had improved to $6 / 9$ and N5, but the right remained at perception of hand movements. Both optic discs now showed temporal pallor. The visual fields (Fig. 5, opposite) showed complete loss of the right field, apart from perception of hand movements in the temporal periphery, and a steep-edged temporal hemianopia in the left. She was therefore again investigated by the neurologists, but the skull $x$ rays and optic foramina were still normal. Electro-encephalography showed no abnormality, but the cerebrospinal fluid showed some increase in total protein and a moderately positive mid-phase colloidal gold curve. With this evidence, supported by visual improvement in the left eye, and the appearance of pallor of the optic discs some 6 months after the initial visual loss, a possible diagnosis of demyelinating disease was suggested with probable plaque formation in the chiasm and right optic nerve. Observation was advised, and no further studies were undertaken.

The patient was seen at regular intervals after this and the position as regards visual acuity and visual fields remained absolutely unaltered from that shown in Fig. 5, but quite marked optic atrophy was apparent by early 1961. The case was extensively reviewed in April, 1963. $X$-ray examinations were repeated and were again negative. It was decided to continue to watch the visual fields carefully and to consider exploration of the chiasmal area to exclude the possibility of a tumour in the event of any further loss in the remaining left nasal field. The patient was instructed to return at once if she noticed any further visual loss. In August, 1963, she was seen for 


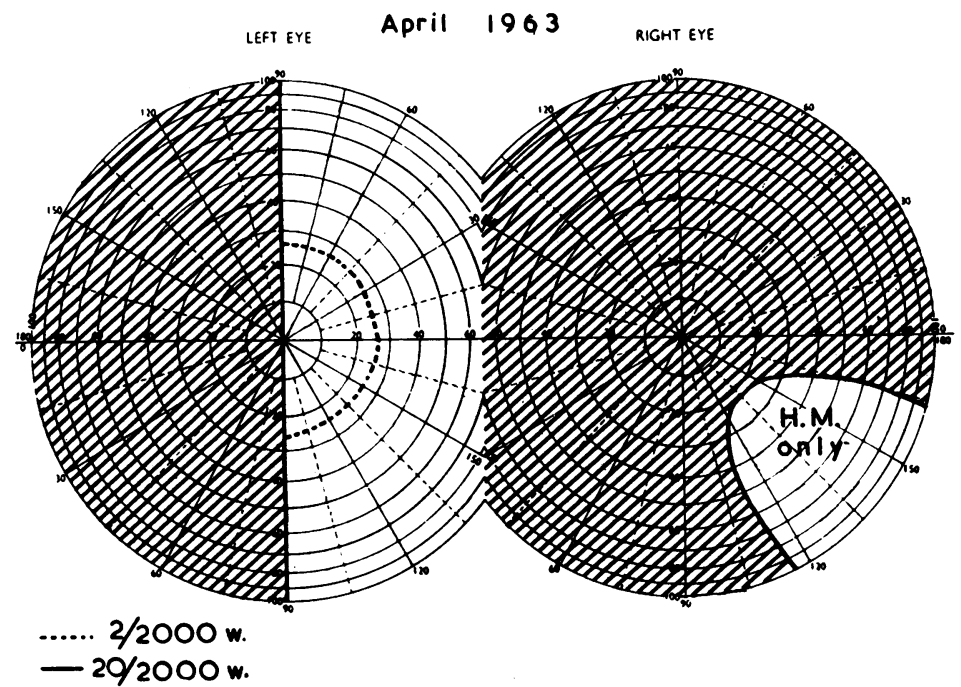

FIG. 5.-Case 2. Visual fields as recorded unchanged from May, 1960, to April, 1963.

Visual acuity: left eye $6 / 9, \mathrm{~N} 5$; right eye, hand movements.

routine follow-up and stated that she had noticed some further loss of vision in the left eye during the previous 2 months, but had not bothered about it. The left visual acuity had dropped to counting fingers, and the visual field was as shown in Fig. 6, a considerable further loss of field in the lower nasal quadrant having taken place. With this evidence of progressive visual loss, the diagnosis of tumour was certain, and the patient was referred to the Neurosurgical Department for further evaluation and possible exploration of the chiasmal region.

$X$ rays of the skull still showed the pituitary fossa to be of normal size, but there was now some erosion of the dorsum sellae and posterior clinoids. Lumbar pneumoencephalography was performed and good visualization of air in the basal cisterns and subarachnoid spaces was obtained. The pictures were reported on as follows by Dr. A. A. Donaldson, Neuroradiologist in the Department of Surgical Neurology:

"There is a deformity of the pontine, interpeduncular, and chiasmatic cisterns, which are filled by a rounded mass apparently arising from the region of the pituitary fossa over the posterior clinoid processes. The optic chiasm appears to be raised, the appearances suggesting a pituitary tumour (see Fig. 7, overleaf). Air in the cistern along the course of the anterior cerebral artery appears normal in distribution, and does not suggest the presence of hydrocephalus. Further investigation by means of angiography would be of interest."

Bilateral common carotid angiography was then performed and reported on as follows:

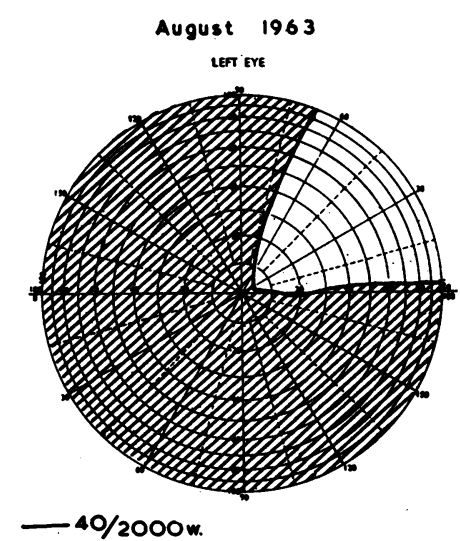

FIG. 6.-Case 2. Left visual field in August, 1963, immediately before operation. Visual acuity: counting fingers.

"On the right side, the appearances are within normal limits.

On the left side there is a little elevation of the proximal portion of the anterior cerebral artery between its origin and the anterior communicating region, and there is a little straightening and lateral deviation of the upper part of the left internal carotid artery compatible with a relatively small supra-sellar mass (Fig. 8, overleaf). No abnormal vascularity is seen in either the arterial or the venous phase films."

Operation.-Exploration of the chiasmal region was therefore performed on September 9, 1963, by Mr. F. J. Shaw through a right fronto-temporal craniotomy. His findings were as follows:

"An extremely pale, thinned, and flattened right optic nerve was exposed. It was also seen to be displaced 


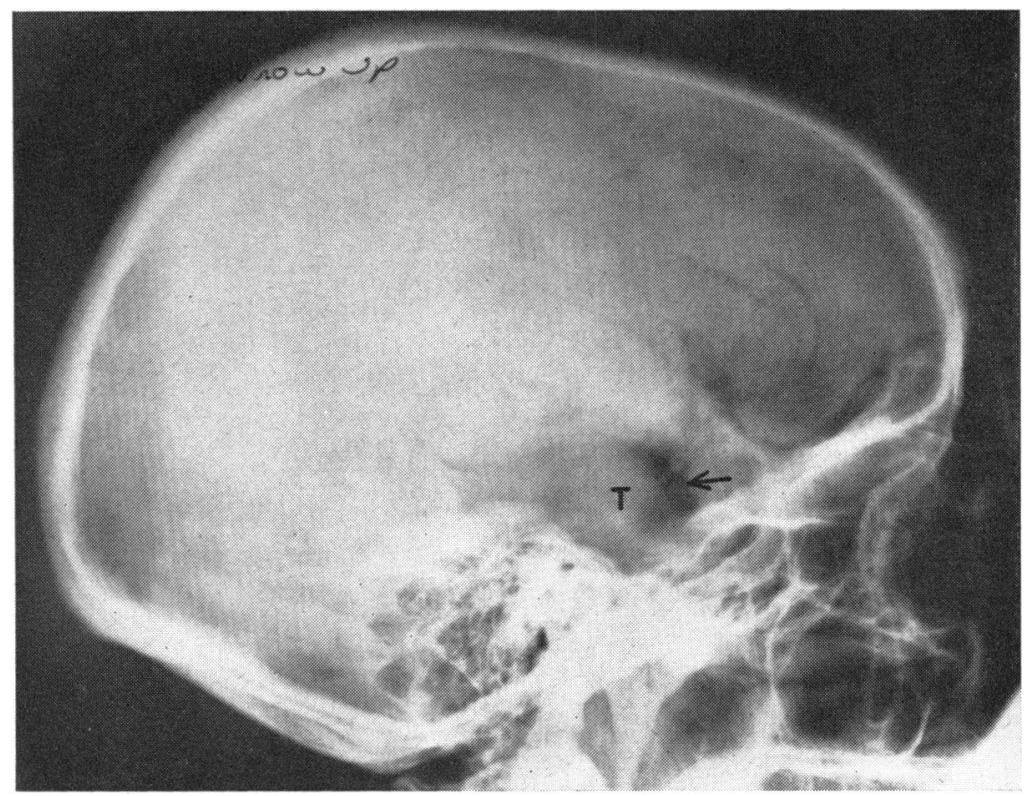

FIG. 7.-Case 2. Lumbar pneumo-encephalogram, showing tumour (T) outlined by air (arrowed) in basal cistern.

Right

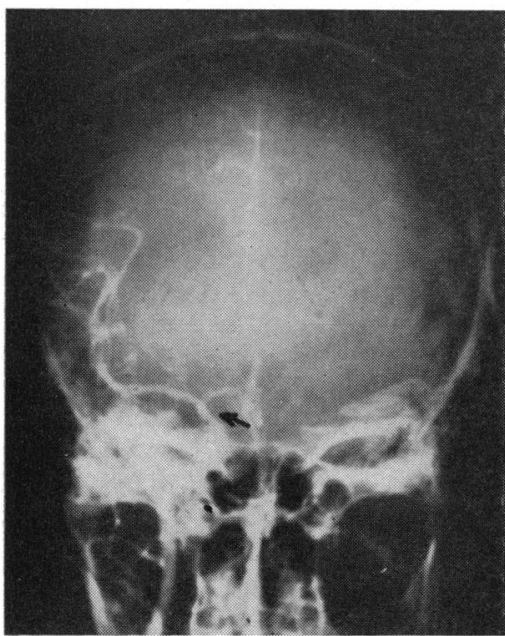

Left

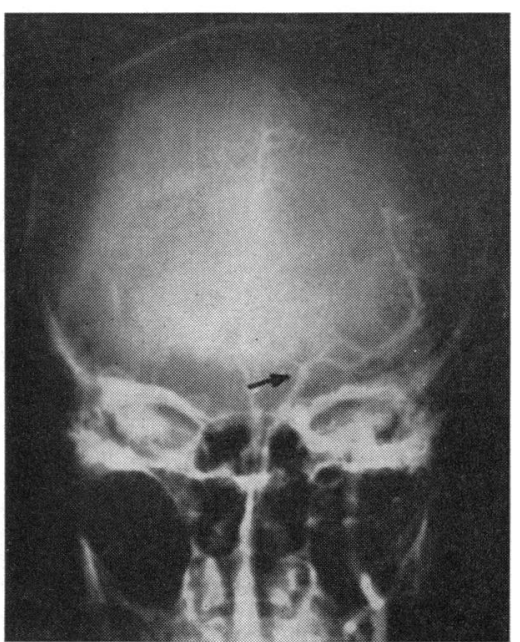

Fig. 8. - Case 2. Bilateral carotid arteriograms, showing minimal elevation of proximal part of left anterior cerebral artery at its origin, and some straightening and lateral deviation of the left internal carotid artery as compared to that on the right.

to the left, this displacement being caused by an underlying cystic lesion with greyish walls. The anterior margin of an equally pale optic chiasm was demonstrated and this was rather prefixed in situation. The right-hand margin of the left optic nerve was also seen. This, though paler than normal, was not so atrophic as the structure on the right side. Having defined the internal carotid artery, a portion of the cyst wall some $1 \frac{3}{4}$ sq. $\mathrm{cm}$. in area was cleared between the right optic nerve and the right internal carotid artery. Because of the rather prefixed situation of the chiasm this was the most easily accessible portion of the cyst wall. Through this the cyst was aspirated with a lumbar puncture needle and $42 \mathrm{cc}$. of straw-coloured rather opalescent fluid were removed. The nature of this fluid suggested an epidermoid tumour. Having emptied 
the cyst of this fluid, a piece of cyst wall was excised and retained for histological examination. It was now possible to inspect the interior of the cyst, which was smooth-walled, without any obvious tumour tissue and a fluctuant bluer portion of its posterior aspect appeared to be due to the adjacent interpeduncular system. There was thus no contained tumour to be removed. Attempts to remove further portions of the cyst wall were unsuccessful, in that it was closely bound to the under surface of the optic nerve and chiasm and similarly bound closely to the internal carotid artery."

Pathological Report on the biopsy specimen:

"Histologically the specimen consists of twisted portions of fibrous tissue and stratified squamous epithelium. No neural or adenohypophyseal tissue is identified. EPIDERMOID CYST (Craniopharyngioma)."

Result.-Post-operative recovery was uneventful and the patient's vision started to recover within the first 2 weeks after operation. Less than 4 weeks after operation on October 2, 1963, the visual field was as shown in Fig. 9, where the peripheral field is seen to have recovered but central vision is still absent. The visual acuity was still only counting fingers. The patient said she had not felt so well for many years.

Comment.-In this case the field defect was identical to that in Case 1 but, in this instance at least in the later stages of observation, optic atrophy was present bilaterally and this was very suggestive of the presence of a tumour in the chiasmal region, despite the history of sudden visual loss. On the other hand, this could have occurred as a result of sudden increase in the size of the tumour, perhaps due to a haemorrhage

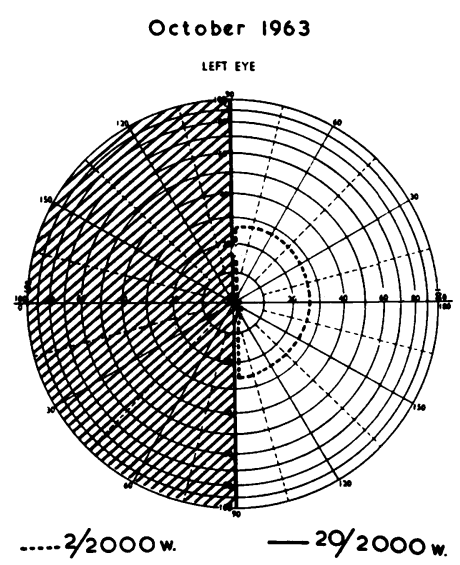

FIG. 9.-Case 2. Left visual field 6 weeks after operation, showing return of peripheral field but persistent loss of fixation. (No further visual improvement had occurred up to the time of publication.) Visual acuity: $2 / 60$. within it. Once again, plain skull $x$ rays did not show anything and arteriography carried out in 1959 was also unrevealing. The progression of the field defect in July-August, 1963, together with the development of confirmatory evidence from the air studies and arteriograms made exploration imperative.

\section{Summary}

Two cases of tumours in the region of the optic chiasm are reported in which the presenting perimetric findings were strongly suggestive of such a lesion, but in which confirmatory evidence was not present on the plain $x$ rays, and angiography was also negative. In each, progressive loss of visual function made it imperative to perform air studies and carry out dye ventriculography in order to confirm the presence of a tumour, when surgical exploration was accordingly carried out.

I am indebted to Prof. G. I. Scott for permission to report these cases and for his advice in the preparation of this paper. I should like to thank Prof. F. J. Gillingham and Mr. J. F. Shaw, who carried out the neurosurgical procedures and made their records available to me. My thanks are also due to Dr. A. A. Donaldson for the neuroradiology and to Dr. A. Gordon for the pathological reports.

\section{REFERENCES}

Alfano, J. E., Almeida, H. C., and Whitworth, C. (1963). Amer. J. Ophthal., 55, 312.

CusHING, H. (1930). Arch. Ophthal. (Chicago), 3, 505.

KeLLY, R. (1962). Trans. ophthal. Soc. U.K., 82, 149.

McConnell, A. A., and MoONEY, A. J. (1938). Brain, 61, 37.

Scort, G. I. (1957). "Traquair's Clinical Perimetry",7th ed., p. 221. Kimpton, London.

WALSH, F. B. (1957). "Clinical Neuro-ophthalmology", 2nd ed., p. 36. Williams and Wilkins, Baltimore. 